\title{
Diagnostic value of 3D-FLAIR magnetic resonance sequence in detection of white matter brain lesions in multiple sclerosis
}

\author{
Ahmed Ibrahim Tawfik ${ }^{*}$ id and Wael Hamza Kamr
}

\begin{abstract}
Background: MS is common demyelinating disease in which standard T2 and 2D-FLAIR MRI sequences play important role in its diagnosis. Recently, 3D-FLAIR sequence is used and has a role that is evaluated compared to standard sequences.

Results: This study was performed on 20 selected MS patients. Brain MRI was performed using routinely used T2 and 2D FLAIR sequences, and 3D-FLAIR sequence was added. 3D-FLAIR images were reformatted, and all images were blindly analyzed. Lesions were counted in each sequence and classified according to their location into supratentorial lesions including periventricular, deep white matter, and juxta-cortical, and infratentorial lesions and relative comparison of lesion number on 3D-FLAIR versus 2D-FLAIR and T2 imaging, respectively, were expressed as percentage gain or a loss.

3D-FLAIR sequence showed significantly more lesions compared to 2D FLAIR and T2 sequences in all locations with relative ratio of $29 \%$ and $41 \%$, respectively, in periventricular region; $22 \%$ and $30 \%$, respectively, in deep WM; $180 \%$ and $147 \%$, respectively, in juxta-cortical region; and $80 \%$ and $13 \%$, respectively, in infratentorial region.
\end{abstract}

Conclusion: 3D-FLAIR sequence is of greater sensitivity than standard 2D-FLAIR and T2 sequences in MS brain lesions depiction, and it is recommended to be included in MR protocol of MS.

Keywords: 3D-FLAIR, Magnetic resonance imaging, Multiple sclerosis

\section{Background}

Multiple sclerosis (MS) is a common auto-immune demyelinating inflammatory disease affecting the central nervous system including brain and spinal cord in which magnetic resonance imaging (MRI) plays an important role in diagnosis. Accordingly, MRI was included in diagnosis in McDonald criteria of 2001 and in their revised versions of 2005 and 2010 as well [1, 2].

MS was considered as a white matter disease for a long time. However, it is proved that it affects also cortical grey matter as well by histopathologic studies [3].

MR imaging has a valuable role in diagnosis and follow-up of MS, as MS lesions formation leads to

\footnotetext{
*Correspondence: ahmedtawfik82@yahoo.com

Diagnostic Radiology Department, Mansoura University, Mansoura, Egypt
}

hydrophilic changes in its site which is reflected as high signal intensity on T2-weighted scans and low signal on T1-weighted scans [4].

Although T2 weight images plays important role in detection of MS lesions, lesion depiction is affected by bright cerebrospinal fluid signal in ventricular system and subarachnoid space that affect lesions in periventricular white matter and juxta-cortical region as both are bright, fluid-attenuated inversion recovery 2D-FLAIR helps to overcome this limitation by suppressing CSF signal and yet creates good contrast between MS lesions and white matter [5].

Unfortunately, great role of 2D-FLAIR in CSF signal suppression leads to reduction of SNR and decrease in contrast between gray matter and white matter especially in old patients. Furthermore, flow artifact seen in

\section{Springer Open}

(๑) The Author(s). 2020 Open Access This article is licensed under a Creative Commons Attribution 4.0 International License, which permits use, sharing, adaptation, distribution and reproduction in any medium or format, as long as you give appropriate credit to the original author(s) and the source, provide a link to the Creative Commons licence, and indicate if changes were made. The images or other third party material in this article are included in the article's Creative Commons licence, unless indicated otherwise in a credit line to the material. If material is not included in the article's Creative Commons licence and your intended use is not permitted by statutory regulation or exceeds the permitted use, you will need to obtain permission directly from the copyright holder. To view a copy of this licence, visit http://creativecommons.org/licenses/by/4.0/. 
Table $1 \mathrm{MRI}$ pulse sequences used in this study

\begin{tabular}{|c|c|c|c|}
\hline & $\mathrm{T} 2$ & 2D-FLAIR & 3D-FLAIR \\
\hline $\begin{array}{l}\text { - TR (ms) } \\
\text { - TE (ms) } \\
\text { - TI (ms) } \\
\text { - Sacn mode } \\
\text { - Slice thickness (mm) } \\
\text { - FOV } \\
\text { - Matrix } \\
\text { - Time (min) }\end{array}$ & $\begin{aligned} & 4840 \\
\cdot & 100 \\
= & - \\
= & 2 \mathrm{D} \\
= & 5 \\
= & 230 \times 185 \times 143 \\
= & 328 \times 198 \\
= & 1: 32\end{aligned}$ & 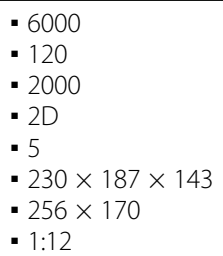 & $\begin{array}{l}=4800 \\
=351 \\
\cdot 1660 \\
\cdot 3 \mathrm{D} \\
=1.5 \\
=250 \times 204 \times 142 \\
\cdot 216 \times 180 \\
\cdot 5: 40\end{array}$ \\
\hline
\end{tabular}

Note: $T R$ repetition time, $T E$ echo time, $T$ inversion time, FOV field of view

2D-FLAIR mainly due to CSF flow and to less extent due to blood flow leads to inadequate T2 weighting [6].

More recently and with evaluation of MRI sequences, three-dimensional MR sequences like 3D-FLAIR have become available with the advantage of high-spatial resolution with high SNR and its capacity to obtain multiplanar reconstruction that allow simultaneous evaluation of the lesion in three orthogonal planes [7, 8], the disadvantage of these three-dimensional sequences is increased acquisition time per scan [9].

This study is focusing on evaluating role of 3D-FLAIR in multiple sclerosis imaging compared to standard T2 and 2D-FLAIR sequences.

\section{Methods}

\section{Patients}

This study was done between March 2017 and October 2018 on 20 selected patients ( 12 females and 8 males) of age ranging from 18 to 45 years and mean age of the patients was 24 years.

Inclusion criteria includes patients clinically diagnosed or suspected to have MS, patients comprised of 14 subjects with relapsing-remitting MS, two of them had acute attacks while others during recovery period and 6 subjects with clinical isolated syndrome (CIS) during their first episode of MS symptoms
Exclusion criteria include patients with concomitant neurological disease in addition to MS and patients with MRI claustrophobia.

This prospective study protocol was approved by the Institutional Review Board and informed consent was obtained from all patients included in the study before MRI acquisition.

\section{MRI imaging}

Brain MRI was performed with a 1.5 Tesla MRI scanner (Ingenia; Philips Medical Systems; Best, The Netherlands) by using an eight-channel head coil. The patients were examined with a standard MS diagnosis protocol including routinely used T2 and 2D-FLAIR sequences and 3D-FLAIR sequence is added in the same session, no post-contrast acquisitions were performed. T2 and 2D-FLAIR were acquired in the axial and coronal planes, and 2D-FLAI $\mathrm{R}$ was acquired in sagittal plane; the slice thickness was $5 \mathrm{~mm}$ in $2 \mathrm{D}$ sequences and $1.5 \mathrm{~mm}$ in $3 \mathrm{D}$-FLAI $\mathrm{R}$. The acquisition time of the $2 \mathrm{D}$ sequences was 1 : 32 and 1:12 min for T2 and 2D-FLAIR sequences, respectively, while it took about 5:40 min with $3 \mathrm{D}$ FLAIR. More detailed sequence parameters are listed in Table 1.

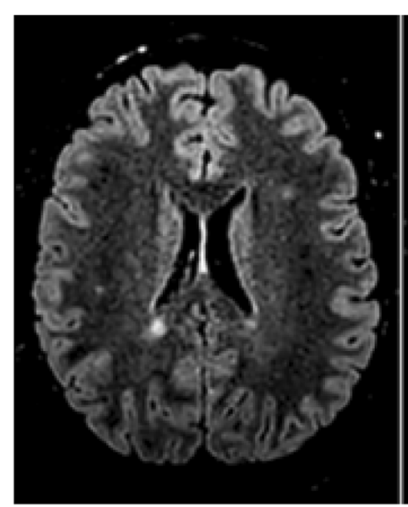

(a)

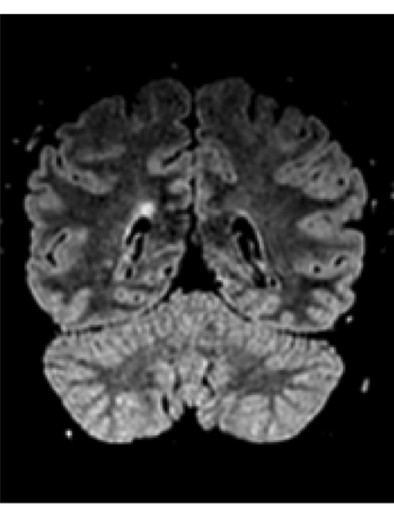

(b)

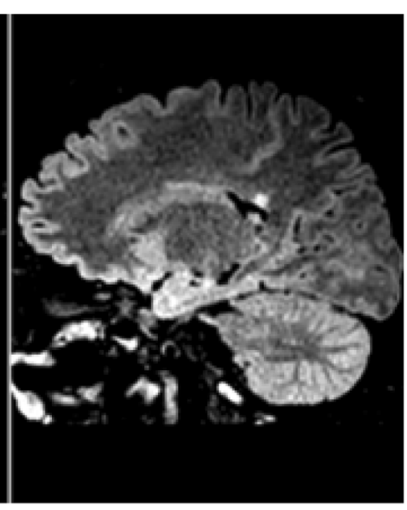

(c)

Fig. 1 Example of multiplanar. a Axial, b coronal, and c sagittal 3D-FLAIR images 
Table 2 Number of detected lesions in all patients in different brain regions by each used sequence and relative comparison of 3DFLAIR versus 2D-FLAIR and T2 pulse sequences

\begin{tabular}{|c|c|c|c|c|c|c|c|c|}
\hline \multirow[t]{2}{*}{ Regions } & & \multirow[b]{2}{*}{ 3D-FLAIRa } & \multirow[b]{2}{*}{ 2D-FLAIRa } & \multirow[b]{2}{*}{ T2a } & \multicolumn{4}{|c|}{ Relative comparison $(\%)^{\mathrm{b}}$} \\
\hline & & & & & 3D-FLAIR/2D-FLAIR & $P$ value $c$ & 3D-FLAIR/T2 & $P$ value $c$ \\
\hline \multirow[t]{3}{*}{ Supra-tentorial } & Periventricular & 107 & 83 & 76 & 29 & $0.001^{*}$ & 41 & $0.001^{*}$ \\
\hline & Deep white matter & 112 & 92 & 86 & 22 & $0.005^{*}$ & 30 & $0.002^{*}$ \\
\hline & Juxta-cortical & 42 & 15 & 17 & 180 & $<0.001^{*}$ & 147 & $0.001^{*}$ \\
\hline Infratentorial & & 9 & 5 & 8 & 80 & 0.28 & 13 & 0.76 \\
\hline Total & & 270 & 195 & 187 & 38 & $<0.001^{*}$ & 44 & $<0.001^{*}$ \\
\hline
\end{tabular}

${ }^{\mathrm{a}}$ Data are numbers of detected lesions

${ }^{b}$ Data are relative differences in the numbers of detected lesions expressed as percentages of lesion numbers identified with 3D-FLAIR imaging compared with the corresponding 2D-FLAIR and T2 imaging

${ }^{c} p$ value was obtained from Wilcoxon analysis for matched pairs indicating that more or fewer patients showed higher lesion load measurement with 3D-FLAIR imaging in comparison with the corresponding 2D-FLAIR or T2 imaging

*Significance $<0.05$

\section{Image analysis}

3D-FLAIR images were reformatted into axial, coronal, and sagittal images (Fig. 1). All T2, 2D-FLAIR images, and 3D-FLAIR images were analyzed by two radiologists who have about 10-year experience in neuroradiology in conjoint reading blinded to patient's clinical presentation. High signal intensity brain lesions with a size of not less than $1 \mathrm{~mm}$ were counted in each of the three pulse sequences, and their number is documented; hyperintense lesion was only counted once when it appeared on multiple contiguous slices. Their location was documented and classified into the following:

(A) Supratentorial lesions which were divided into the following:
1. Periventricular lesions (those lesions abutting lateral ventricles and rarely third ventricle).

2. Deep white matter lesions (in deep white matter).

3. Juxta-cortical lesions (within the subcortical white matter immediately adjacent to the grey matter).

(B) Infratentorial lesions (located in or along the surface of the cerebellum and brain stem).

\section{Statistical analysis}

For data analysis Statistical Package for Social Science software computer program version 23 (SPSS, Inc., Chicago, IL, USA) was used. Analysis of multiple brain lesions on the different pulse sequences (3DFLAR, 2D-FLAIR, and T2) was performed. The statistical differences in analyses were assessed using the

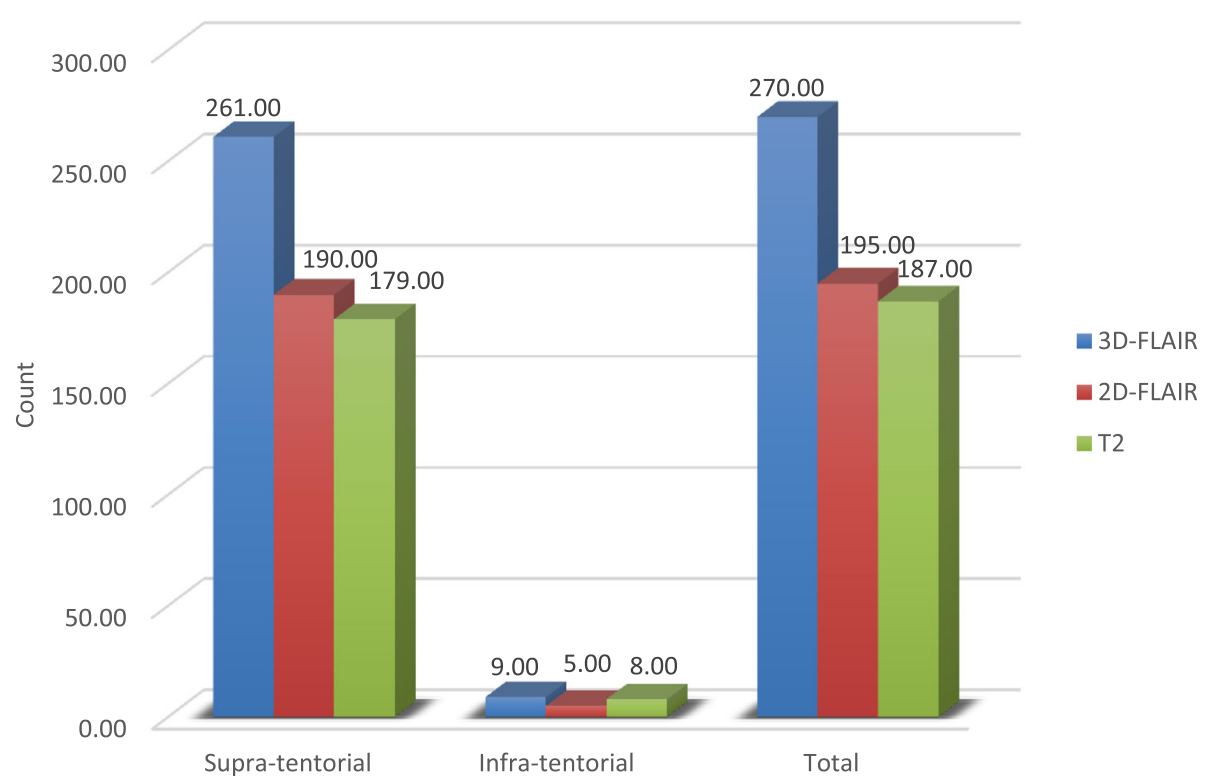

Fig. 2 Analysis of the lesion load detection by 3D-FLAIR, 2D-FLAIR, and T2WI according to the total number of lesions in the supratentorial, infratentorial regions and in both regions 


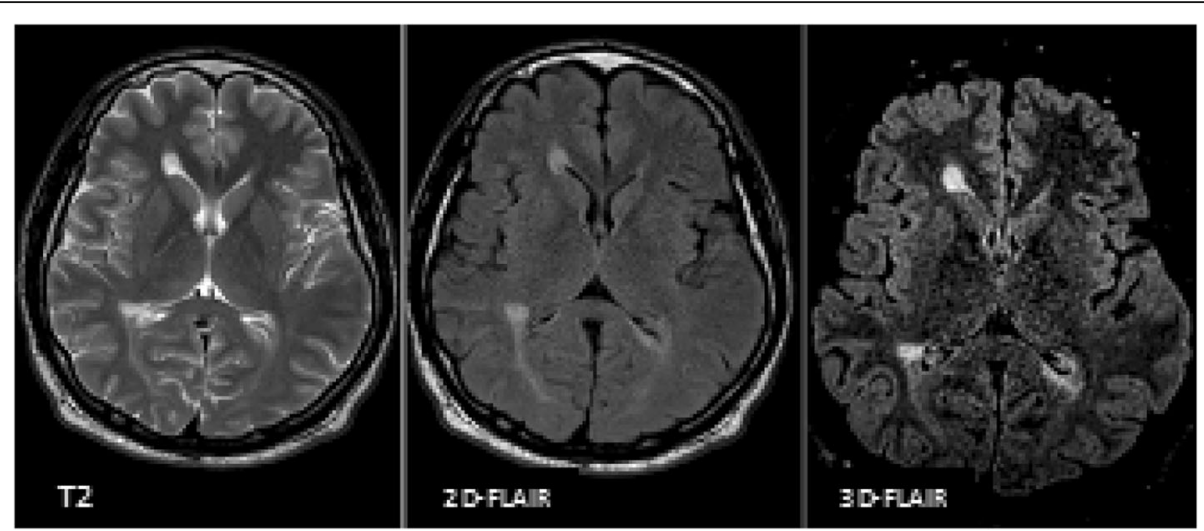

Fig. 3 Axial T2, 2D FLAIR, and 3D-FLAIR images showing periventricular white matter more clearly distinguished on 3D-FLAIR than T2 and 2D-FLAIR images

Wilcoxon test for matched pairs. The relative comparison of the number of MS brain lesions on 3DFLAIR versus 2D-FLAIR and T2 imaging, respectively, was expressed as percentage gain or a loss in the number of detected brain lesions. All calculations were performed by the SPSS software package (SPSS,
Chicago, IL, USA). $P$ value less than 0.05 was considered statistically significant.

\section{Results}

3D-FLAIR sequence revealed 270 lesions representing the overall load measurement (the total number of

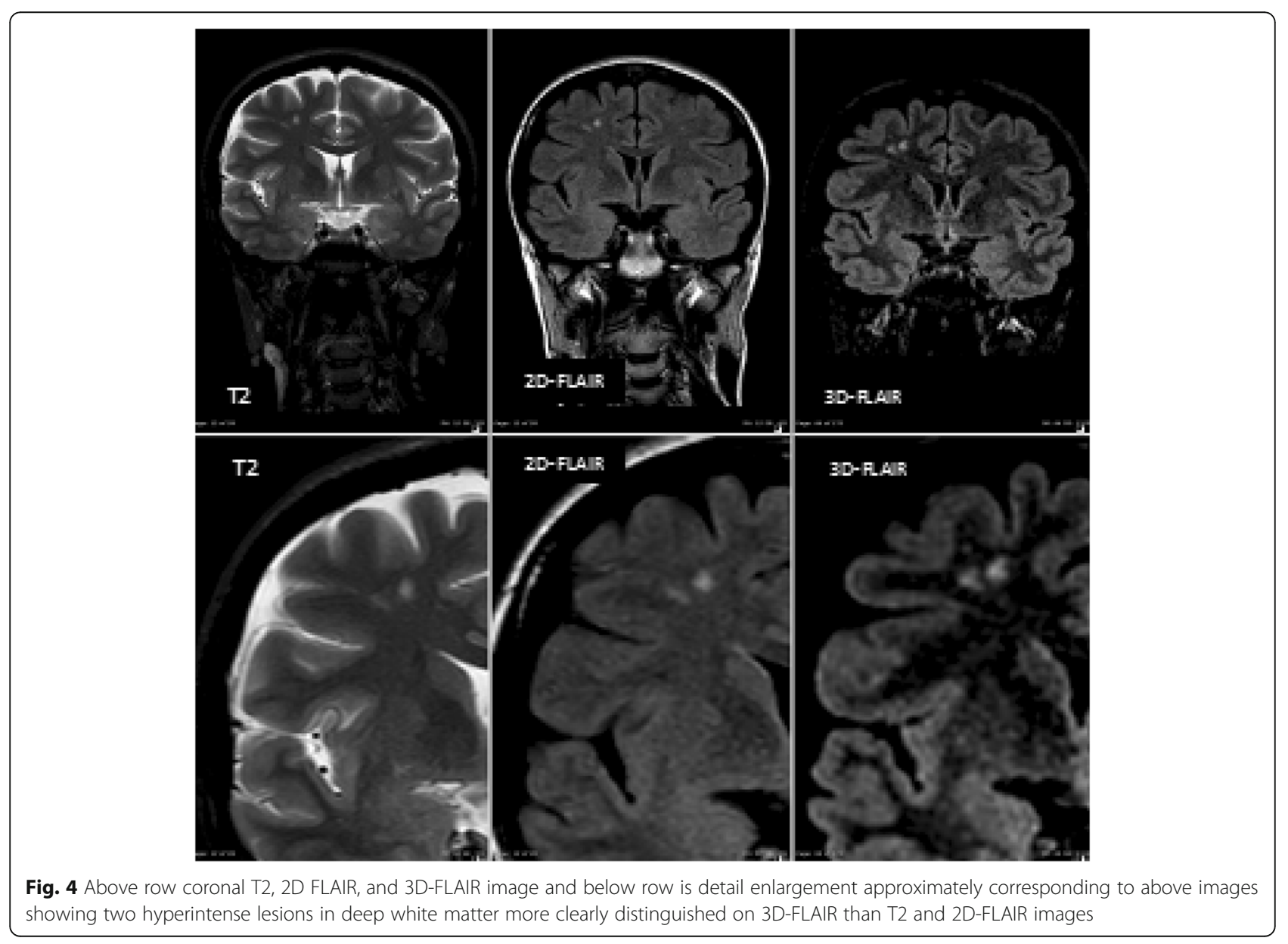




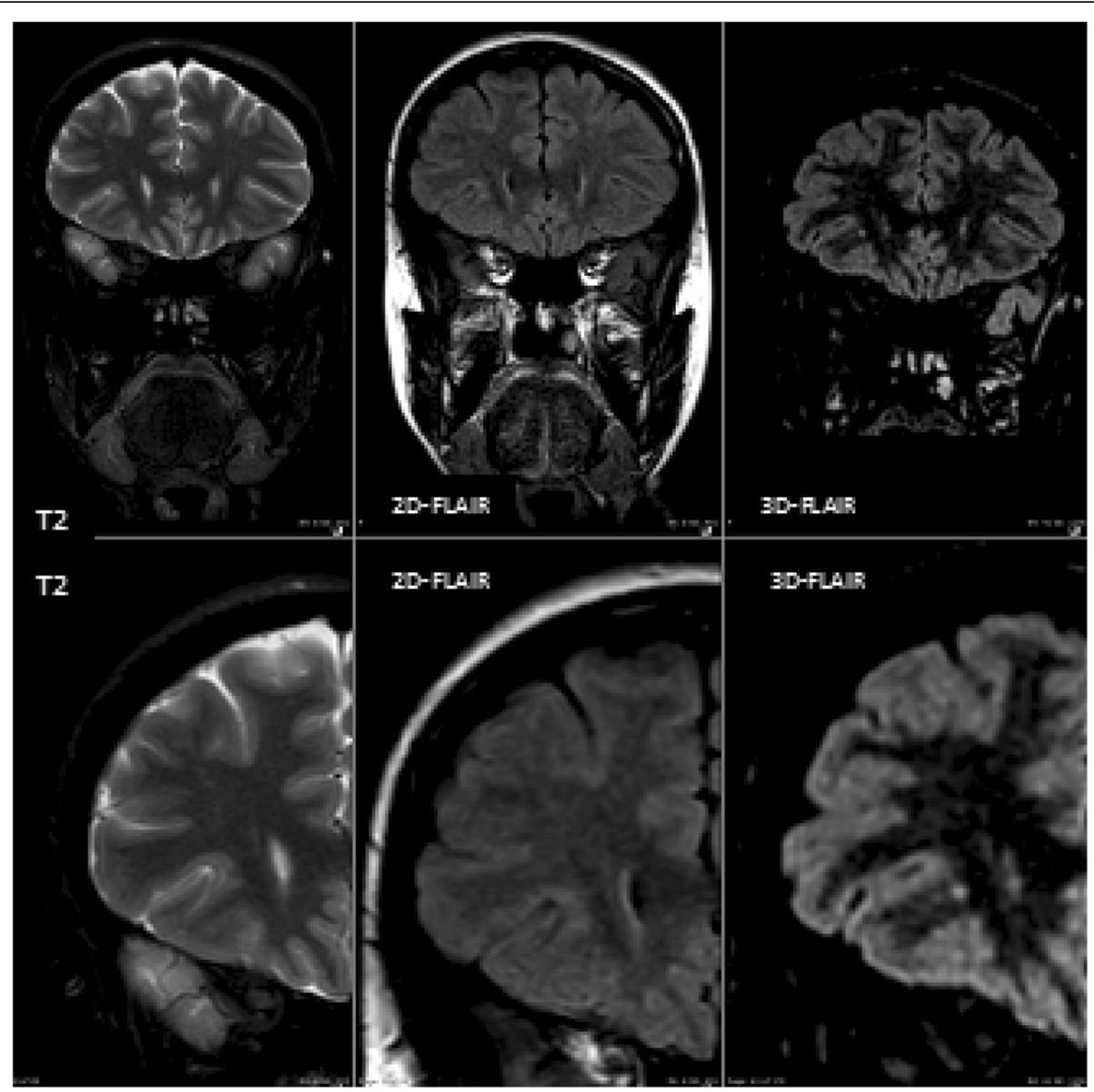

Fig. 5 Above row coronal T2, 2D FLAIR, and 3D-FLAIR image and below row is detail enlargement approximately corresponding to above images in the same patient of Fig. 3 showing single small hyperintense lesions seen in juxta-cortical white matter seen on 3D-FLAIR and not depicted on T2 and 2D-FLAIR images

lesions in all cases), while 2D-FLAIR sequence revealed 195 lesions and T2 pulse sequence revealed 187 lesions. (Table 2, Fig. 2)

3D-FLAIR sequence was significantly superior to T2 (P less than 0.001 with a relative ratio of $44 \%$ ) and significantly superior to 2D-FLAIR ( $P$ less than 0.001 with a relative ratio of $38 \%$ ) regarding the total load measurement (Table 2).

In supratentorial region, total number of lesions depicted by 3D-FLAIR 261 lesions, 190 lesions by 2DFLAIR sequence, and 179 lesions by T2 pulse sequence (Table 2, Fig. 2)

Supratentorial lesions were further categorized according to their anatomical location into (periventricular WM, deep WM, and juxta-cortical WM).

In periventricular region (Fig. 3), the total number of lesions was depicted by 3D-FLAIR 107 lesions: 83 lesions by 2D-FLAIR sequence and 76 lesions by T2 pulse sequence in deep white matter region (Fig. 4); the total number of lesion depicted by 3D-FLAIR 112 lesions: 92 lesions by 2D-FLAIR sequence and 86 lesions by T2 pulse sequence; finally, in juxta-cortical region (Figs. 5 and 6), the total number of lesions depicted by 3D-FLAI R 42 lesions: 15 lesions by 2D-FLAIR sequence and 17 lesions by $\mathrm{T} 2$ pulse sequence (Table 2, Fig. 7).

3D-FLAIR sequence was superior to T2 WM and FLAIR sequence in lesion detection in all these locations as detailed.

In periventricular WM region, 3D-FLAIR sequence showed significantly more lesions compared to 2DFLAIR sequence $(P=0.001$ with a relative ratio of $29 \%)$, and compared to T2 $(P=0.001$ with a relative ratio of $41 \%$ ), in deep WM 3D-FLAIR sequence showed significantly more lesions compared to T2W sequence $(P=0.001$ with a relative ratio of $30 \%)$ and compared to 2D-FLAIR sequence $(P=0.005$ with a relative ratio of $22 \%$ ) and in juxta-cortical region 3DFLAIR sequence showed significantly more lesions compared to the T2W sequence $(P=0.001$ with a relative ratio of $147 \%$ ) and compared to 2D-FLAIR sequence $(P$ less than 0.001 with a relative ratio of 180\%) (Table 2). 

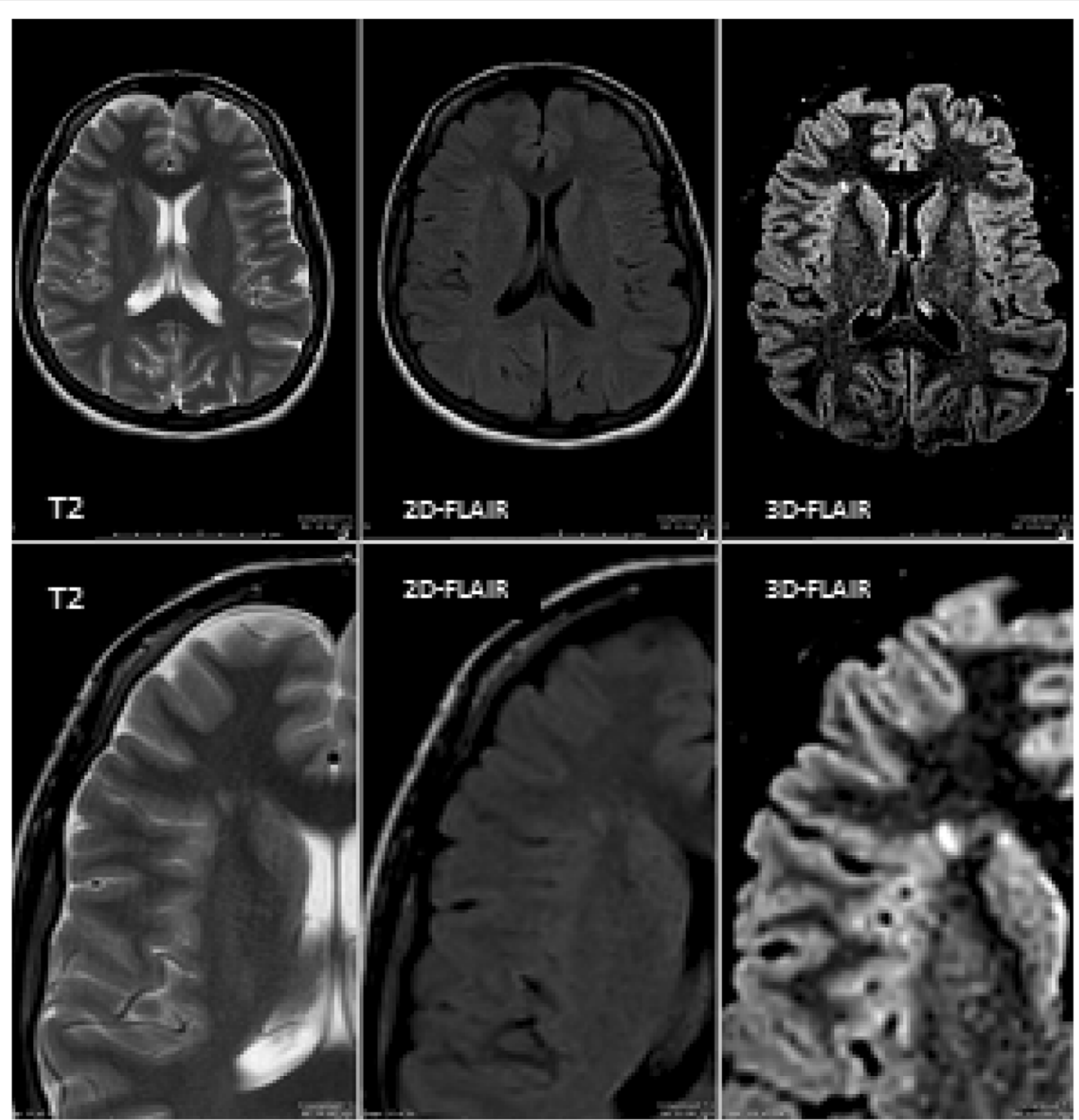

Fig. 6 Above row axial T2, 2D FLAIR, and 3D-FLAIR image and below row is detail enlargement approximately corresponding to above images showing single hyperintense lesion seen in juxta-cortical white matter; it is better seen on 3D-FLAIR images with good contrast to both white and grey matter and less depicted on T2 and 2D-FLAIR images

In infratentorial region (Fig. 8), 3D-FLAIR sequence revealed 9 lesions, while 2D-FLAIR sequence revealed 5 lesions and T2 pulse sequence revealed 8 lesions (Table 2, Fig. 2).

3D-FLAIR sequence was superior to T2 sequence in depicting the infratentorial lesions with a relative ratio of $13 \%$ and also superior to 2D-FLAIR sequence with a relative ratio of $80 \%$ (Table 2).

\section{Discussion}

3D MRI sequences have many advantages over 2D sequences. Images acquired by 3D sequences have isotropic voxel dimensions and absence of inter-slice gap so it can be easily reformatted without degrading the image quality; in addition, it allows time acceleration using compressed sensing [10]. Another good factor in 3D images is decreased slice thickness that also leads to increased number of detected lesions [11].
Early studies showed that 3D-FLAIR is more superior than routinely used 2D sequences (T2 and 2D-FLAIR) in detection of MS lesions [12, 13]; however, in early trials, $3 \mathrm{D}$ sequences took long acquisition times as it were multi-slab mode, and that disadvantage was adjusted by using single-slab mode [14].

In this study, although 3D-FLAIR sequence has longer time 5:40 min compared to 1:32 $\mathrm{min}$ in $\mathrm{T} 2$ sequence and 1:12 min in 2D FLAIR sequence, it is not considered a drawback as it is 3D volume images by which axial, coronal, and sagittal reformate images could be obtained.

3D-FLAIR sequence is one of 3D sequences that have advantage of good signal with small voxels and by turn high SNRs $[9,15]$. Another advantage seen in 3D-FLAIR sequences is better CSF suppression than T2 and 2D FLAIR sequences and by turn absence of CSF flow artifacts that occurs because large volume 


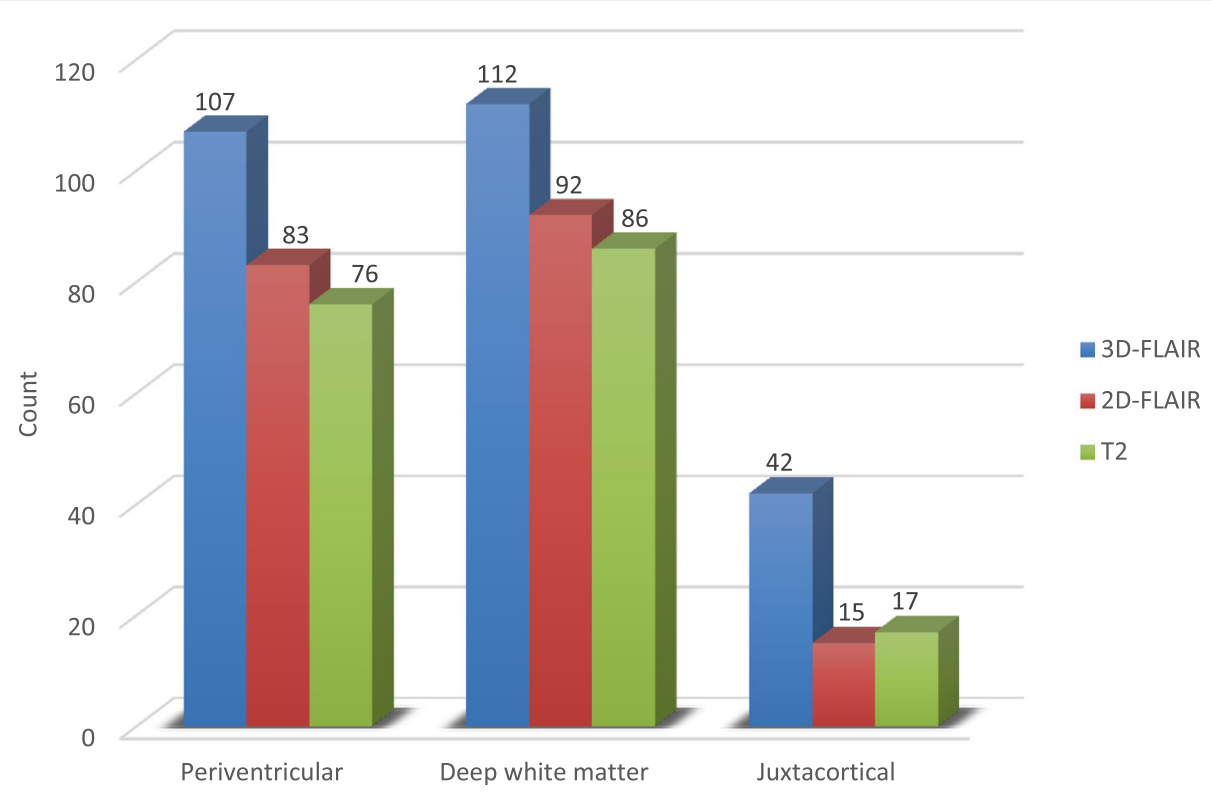

Fig. 7 Analysis of supratentorial white matter lesion detection by 3D-FLAIR, 2D-FLAIR, and T2WI according to their anatomical site (periventricular, deep white matter, and juxta-cortical).

excited using 3D technique; these advantages improve MS lesion detection [16].

In current study, 3D-FLAIR sequence significantly detected more overall number of lesions comparing to standard used 2D-FLAIR and T2 sequences.

In current study, the detected lesions were mainly in supratentorial region (periventricular, deep WM and juxta-cortical), and in all three locations, 3DFLAIR sequence showed significantly more lesions than 2D-FLAIR and T2 sequences. We found that in juxta-cortical region, much more lesions were depicted by 3D-FLAIR sequence than 2D-FLAIR and T2 sequences with more significance and highpercentage ratio than in periventricular WM and deep WM. The major number of that higher count of lesions was due to depiction of new smaller lesions not seen by 2D-FLAIR and T2 sequences, in addition to many lesions that appeared as single confluenting lesion on both sequences were found to be multiple distinct lesions adjacent to each other by 3D-FLAIR.

Recently, juxta-cortical lesions more frequently occur and have found to be related to more clinical disability [17-19].

Few lesions seen in infratentorial region in this study is probably due to limited number of patients, that is keeping with Patzig M et al. [20] that reported few infratentorial lesions found in brain stem and cerebellum probably due to limited number of patients that was also seen in our study.

In current present study, 3D-FLAIR sequence was superior to $\mathrm{T} 2$ sequence in depicting the infratentorial lesions and also much more superior to 2D-FLAIR sequence.

It have been found that 2D-FLAIR sequences are not ideal in the depiction of infratentorial lesion due to CSF flow artifact and lower contrast seen between lesion and white matter [21, 22].

This study results are in keeping with Moraal B et al. [9] who reported that 3D-FLAIR sequence provided the highest sensitivity for depiction of hyperintense MS plaque in all anatomical locations including supratentorial and infratentorial lesions compared to any other 2D sequences, in particular 2D-T2SE, that could lead to earlier diagnosis of the disease.

In MS, there is associated changes white matter diffusion seen in diffusion-weighted images (DWI), and it index apparent diffusion coefficient (ADC) by primary inflammatory changes consisting of the cytotoxic edema followed by vasogenic edema, and active plaques may demonstrate high or low ADC (increased or decreased diffusion) [23].

Larsson HB et al. [24] declares the role of DWI in early depiction of acute MS lesions, and few other studies have discussed the role of diffusion as good diagnostic method as comparable to conventional MRI with conflicting results $[24,25]$

Many studies have been done and examined the role of 3D DIR sequences in diagnosis of MS; in DIR sequences, there is using of additional inversion pulse that increase attenuation of white matter and CSF, and in turn, increase lesion hyperintensity and its depiction; 3D-FLAIR sequences stimulates similar effect 


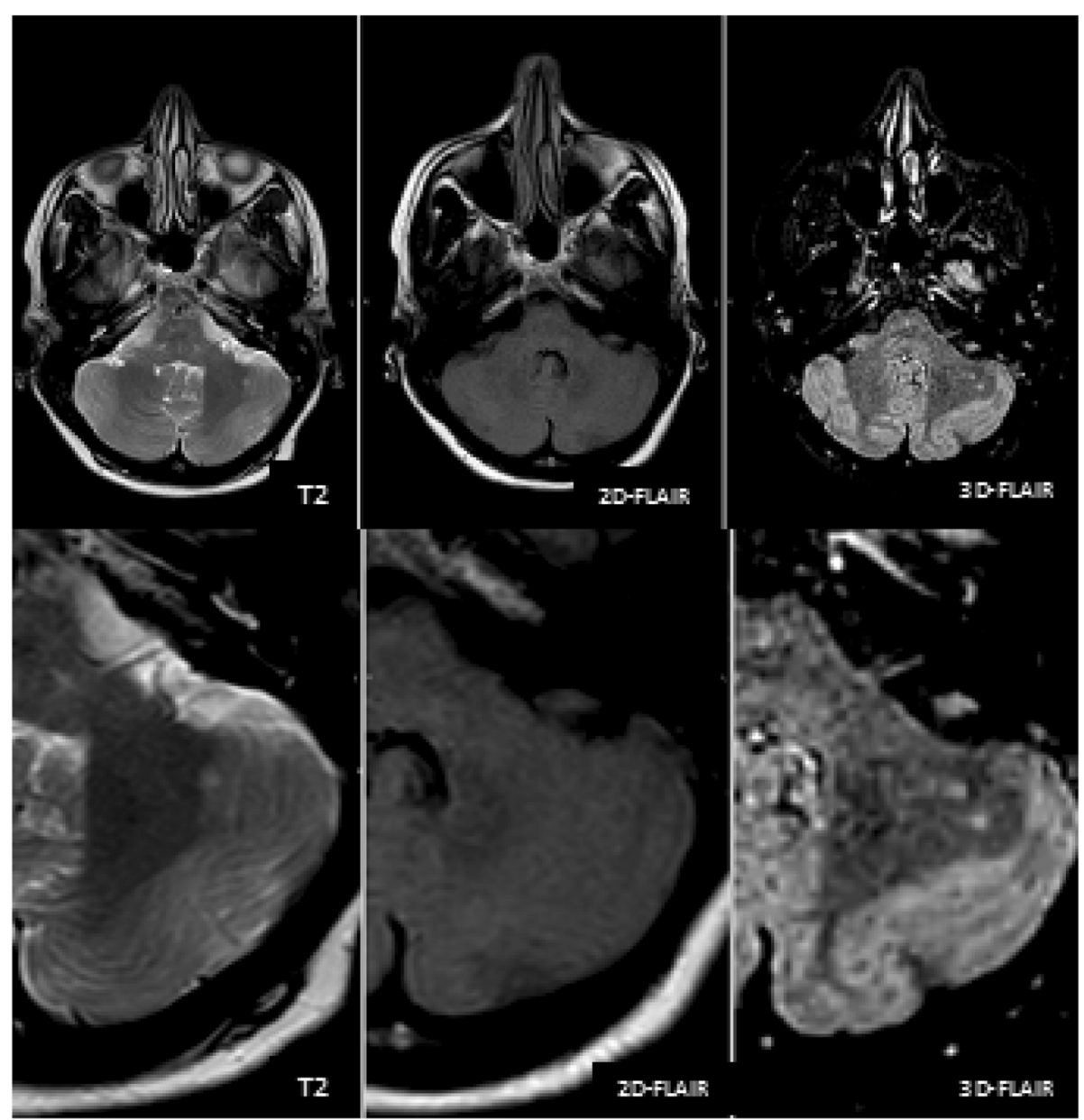

Fig. 8 Above row axial T2, 2D FLAIR, and 3D-FLAIR image and below row is detail enlargement approximately corresponding to above images showing two hyperintense lesions in left cerebellar hemisphere that appear well on 3D-FLAIR than T2 images in which only the larger lesion appears and the smaller one hardly seen and 2D-FLAIR images in which both lesions are not well depicted

and white matter lesions become more hyperintense $[9,26,27]$.

Further studies are needed to compare results of 3DFLAIR and 3D-DIR in depiction of MS lesions.

Acknowledgement of study limitation due to limited number of MS patients is included in the study, but statistical analysis from this work was based on large total number of MS lesions which was considered sufficient.

\section{Conclusion}

3D-FLAIR sequence is valuable in diagnosis and followup of brain MS lesions as it can detect much more lesions as compared to the standard routinely used 2DFLAIR and T2 sequences in brain supratentorial and infratentorial regions. 3D-FLAIR sequences showed better depiction and better delineation between the lesions and both white and grey matters; we recommend 3DFLAIR sequence to be included in routine MR protocols of MS patients.

\section{Abbreviations}

2D-FLAIR: Two-dimensional-fluid-attenuated inversion recovery; 3D-FLAI R: Three-dimensional-fluid-attenuated inversion recovery; 3D-DIR: Threedimensional-double inversion recovery; CSF: Cerebrospinal fluid; FOV: Field of view; MRI: Magnetic resonance imaging; MS: Multiple sclerosis; TE: Echo time; TI: Inversion time; TR: Repetition time

\section{Acknowledgements}

Not applicable

\section{Authors' contributions}

WK contributed to the study concepts, quality control of data and algorithms, and data analysis and interpretation. AT contributed to the study design, data acquisition, manuscript preparation, and manuscript editing. AT and WK contributed to the statistical analysis and manuscript review. All authors have read and approved the manuscript.

\section{Funding}

Not applicable

Availability of data and materials

The datasets used and analyzed during the current study are available from the corresponding author on reasonable request. 


\section{Ethics approval and consent to participate}

This study was approved by Research Ethics Committee of Almana General Hospital, Saudi Arabia, on 8/2/2017 (reference number not available). All patients included in this study (all above 16 years old) gave written informed consent to participate in this research.

\section{Consent for publication}

All patients included in this research (all above 16 years old) gave written informed consent to publish the data contained within this study.

\section{Competing interests}

The authors declare that they have no known competing financial interests or personal relationships that could have appeared to influence the work reported in this paper.

Received: 21 January 2020 Accepted: 8 July 2020

Published online: 17 July 2020

\section{References}

1. Polman CH, Reingold SC, Banwell B et al (2011) Diagnostic criteria for multiple sclerosis: 2010 revisions to the "McDonald criteria.". Ann Neurol 69: 292-302

2. Polman CH, Wolinsky JS, Reingold SC (2005) Multiple sclerosis diagnostic criteria: three years later. Mult Scler 11:5-12

3. Kidd D, Barkhof F, McConnell R et al (1999) Cortical lesions in multiple sclerosis. Brain 122(pt 1):17-26

4. Stewart WA, Hall LD, Berry K et al (1984) Correlation between NMR scan and brain slice data in multiple sclerosis. Lancet 2:412 Medline

5. Traboulsee A, Li DK (2008) Conventional MR imaging. Neuroimaging Clin N Am 18:651-673 x CrossRef Medline

6. Okuda T, Korogi Y, Shigematsu Y et al (1999) Brain lesions: when should fluid-attenuated inversion-recovery sequences be used in MR evaluation. Radiology 212:793-798 CrossRef Medline

7. Brau AC, Beatty PJ, Skare S et al (2008) Comparison of reconstruction accuracy and efficiency among autocalibrating data-driven parallel imaging methods. Magn Reson Med 59:382-395

8. Lum DP, Busse RF, Francois CJ et al (2009) Increased volume of coverage for abdominal contrast-enhanced MR angiography with two-dimensional autocalibrating parallel imaging: initial experience at 3.0 Tesla. J Magn Reson Imaging 30:1093-1100

9. Moraal B, Roosendaal SD, Pouwels PJ et al (2008) Multi-contrast, isotropic, single-slab 3D MR imaging in multiple sclerosis. Eur Radiol 18:2311-2320 CrossRef Medline

10. Toledano-Massiah S, Sayadi A, Boer R et al (2018) Accuracy of the compressed sensing accelerated 3D-FLAIR sequence for the detection of MS plaques at 3T. AJNR 39:454-458 Crossref. PubMed

11. Dolezal O, Dwyer MG, Horakova D et al (2007) Detection of cortical lesions is dependent on choice of slice thickness in patients with multiple sclerosis. Int Rev Neurobiol 79:475-489

12. Tubridy N, Barker GJ, Macmanus DG et al (1998) Three-dimensional fast fluid attenuated inversion recovery (3D fast FLAIR): a new MRI sequence which increases the detectable cerebral lesion load in multiple sclerosis. Br J Radiol 71:840-845

13. Tan IL, Pouwels PJ, van Schijndel RA et al (2002) Isotropic 3D fast FLAIR imaging of the brain in multiple sclerosis patients: initial experience. Eur Radiol 12:559-567

14. Mugler JP 3rd, Bao S, Mulkern RV et al (2000) Optimized single-slab threedimensional spin-echo MR imaging of the brain. Radiology 216:891-899

15. Bink A, Schmitt M, Gaa J et al (2006) Detection of lesions in multiple sclerosis by 2D FLAIR and single-slab 3D FLAIR sequences at 3.0 T: initial results. Eur Radiol 16:1104-1110

16. Lummel $N$, Schoepf $V$, Burke $M$ et al (2011) 3D fluid-attenuated inversion recovery imaging: reduced CSF artifacts and enhanced sensitivity and specificity for subarachnoid hemorrhage. AJNR Am J Neuroradiol 32:20542060

17. Bo L, Geurts JJ, Mork SJ et al (2006) Grey matter pathology in multiple sclerosis. Acta Neurol Scand Suppl 183:48-50

18. Kutzelnigg A, Lassmann $H$ (2006) Cortical demyelination in multiple sclerosis: a substrate for cognitive deficits? J Neurol Sci 245:123-126
19. Kutzelnigg A, Lucchinetti CF, Stadelmann C et al (2005) Cortical demyelination and diffuse white matter injury in multiple sclerosis. Brain 128:2705-2712

20. Patzig M et al (2014) Comparison of 3D Cube FLAIR with 2D FLAIR for Multiple Sclerosis Imaging at 3 Tesla. Neuroradiology 186(05):484-488

21. McGowan JC, Patel RS (2000) Technical issues for MRI examination of the posterior fossa. J Neurol Sci 172(Suppl 1):S40-S42

22. Stevenson VL, Parker GJ, Barker GJ et al (2000) Variations in T1 and T2 relaxation times of normal appearing white matter and lesions in multiple sclerosis. J Neurol Sci 178:81-87

23. Yurtsever I, Hakyemez B, Taskapilioglu O, Erdogan C, Turan OF, Parlak M (2008) The contribution of diffusion-weighted MR imaging in multiple sclerosis during acute attack. Eur J Radiol 65(3):421-426

24. Larsson HB, Thomsen C, Frederiksen J, Stubgaard M, Henriksen O (1992) In vivo magnetic resonance diffusion measurement in the brain of patients with multiple sclerosis. Magn Reson Imaging 10(1):7-12

25. Lo CP, Kao HW, Chen SY, Chu CM, Hsu CC, Chen YC et al (2014) Comparison of diffusion-weighted imaging and contrast-enhanced T1weighted imaging on a single baseline MRI for demonstrating dissemination in time inmultiple sclerosis. BMC Neurol 14:100

26. Geurts JJ, Pouwels PJ, Uitdehaag BM, Polman CH, Barkhof F, Castelijns JA (2005) Intracortical lesions in multiple sclerosis: improved detection with 3D double inversion-recovery MR imaging. Radiology 236:254-260

27. Wattjes MP, Lutterbey GG, Gieseke J, Traber F, Klotz L, Schmidt S, Schild HH (2007) Double inversion recovery brain imaging at 3T: diagnostic value in the detection of multiple sclerosis lesions. AJNR Am J Neuroradiol 28:54-59

\section{Publisher's Note}

Springer Nature remains neutral with regard to jurisdictional claims in published maps and institutional affiliations.

\section{Submit your manuscript to a SpringerOpen ${ }^{\bullet}$ journal and benefit from:}

- Convenient online submission

- Rigorous peer review

- Open access: articles freely available online

High visibility within the field

- Retaining the copyright to your article

Submit your next manuscript at $>$ springeropen.com 